\title{
Drink alcohol and dim the lights: The impact of cognitive deficits on medial frontal cortex function
}

\author{
NiCK YeUNG \\ University of Oxford, Oxford, England \\ and Carnegie Mellon University, Pittsburgh, Pennsylvania \\ JASON RALPH \\ Carnegie Mellon University, Pittsburgh, Pennsylvania \\ AND \\ SANDER NIEUWENHUIS \\ Leiden University, Leiden, The Netherlands
}

\begin{abstract}
Scalp electroencephalogram (EEG) recordings indicate that regions in the medial frontal cortex (MFC) are active following errors. Alcohol consumption reduces this error-related activity, perhaps suggesting that alcohol disrupts the operation of an error-monitoring system in the MFC. However, it could also be that alcohol consumption affects the MFC only indirectly, by impairing stimulus processing and thus making errors harder to detect. This interpretation leads to the prediction that stimulus degradation should likewise reduce error-related activity in the MFC. To test this hypothesis, behavioral and EEG data were collected as participants performed a speeded response task with either bright or very dim stimuli. The results using dim stimuli replicated the observed effects of alcohol consumption - with slowed responses accompanied by reduced error-related MFC activity. The sensitivity of the MFC to disrupted processing elsewhere in the brain suggests complications in interpreting evidence of disturbed MFC function.
\end{abstract}

Normal cognitive functioning depends critically on our ability to monitor our thoughts and actions so that we can identify situations in which we need to try harder or try something different (i.e., when increased cognitive control is required). Neuroimaging studies have consistently implicated regions in the medial frontal cortex (MFC), particularly the dorsal anterior cingulate cortex and the presupplementary motor area, in this monitoring process. Thus, MFC activity is observed when participants make errors (Falkenstein, Hohnsbein, Hoorman, \& Blanke, 1990; Gehring, Goss, Coles, Meyer, \& Donchin, 1993) and when conditions of response uncertainty, or conflict, make errors likely (Botvinick, Nystrom, Fissell, Carter, \& Cohen, 1999; Carter et al., 1998). Findings such as these have led to the suggestion that the MFC is involved in monitoring ongoing performance in order to signal the need for increased cognitive control.

Scalp electroencephalogram (EEG) recordings have identified event-related brain potential correlates of the proposed MFC monitoring functions. MFC activity following errors is evident in EEG recordings as the errorrelated negativity (ERN), a frontocentral component that peaks within $100 \mathrm{msec}$ of an incorrect response (Falk- enstein et al., 1990; Gehring et al., 1993). Meanwhile, conflict-related activity in the MFC is reflected in a second EEG component, labeled the N2, that shares the frontocentral scalp distribution of the ERN (Kopp, Rist, \& Mattler, 1996; Nieuwenhuis, Yeung, van den Wildenberg, \& Ridderinkhof, 2003; van Veen \& Carter, 2002; Yeung, Botvinick, \& Cohen, 2004). Together, the ERN and N2 provide a window on MFC function, and a good deal of research has focused on identifying the cognitive and neural basis of these components.

One fruitful line of research has been the use of the ERN and N2 to investigate how MFC function is affected by various neurological disturbances. To this end, the two components have been studied in various clinical populations, including individuals with schizophrenia (Alain, McNeely, He, Christensen, \& West, 2002; Kopp \& Rist, 1999; McNeely, West, Christensen, \& Alain, 2003), borderline personality disorder (Ruchsow et al., 2006), depression (Hajcak, McDonald, \& Simons, 2004; Luu, Collins, \& Tucker, 2000), anxiety disorder (Hajcak, McDonald, \& Simons, 2003), and obsessive-compulsive disorder (Gehring, Himle, \& Nisenson, 2000; Nieuwenhuis, Nielen, Mol, Hajcak, \& Veltman, 2005). The ERN and

N.Yeung, nicholas.yeung@psy.ox.ac.uk 
N2 have also been used to investigate the cognitive impact of alcohol (Curtin \& Fairchild, 2003; Ridderinkhof et al., 2002), caffeine (Tieges, Ridderinkhof, Snel, \& Kok, 2004), and other psychoactive drugs (de Bruijn, Hulstijn, Verkes, Ruigt, \& Sabbe, 2004; Riba, Rodriguez-Fornells, Morte, Munte, \& Barbanoj, 2005; Zirnheld et al., 2004). Taken together, the available evidence paints a clear picture of an MFC monitoring system that is sensitive to a wide range of neurological disturbances.

Observations of disturbed MFC function are potentially very informative about the nature of processing in this region and, conversely, about the cognitive deficits apparent in clinical populations. However, observations of altered MFC function in the context of cognitive and behavioral deficits do not directly establish the direction of causality involved: MFC disruption could be the cause of the observed symptoms, but it could equally well be that this disruption is itself a knock-on effect of primary impairments occurring elsewhere. The MFC shares anatomical connections with a widely distributed network of cortical and subcortical regions (Croxson et al., 2005; van Hoesen, Morecraft, \& Vogt, 1993), and normal MFC functioning presumably depends critically on its interactions with these regions. Damage to any part of this interconnected system would therefore be expected to have some impact on MFC functioning. Consistent with this supposition, focal lesions outside of the MFC have consistently been found to influence the functioning of this region as it is reflected in the ERN and N2 (e.g., Gehring \& Knight, 2000; Ullsperger \& von Cramon, 2006; Ullsperger, von Cramon, $\&$ Muller, 2002).

Where MFC disturbances are observed in patient groups or following drug administration, it is therefore crucial to establish whether these disturbances are a primary cause of the symptoms observed or whether they are, rather, a secondary effect of damage elsewhere. In previous work, we have used computational models as a tool for drawing causal inferences of this kind (Yeung \& Cohen, 2006). This work led us to a new account of Ridderinkhof et al.'s (2002) finding that alcohol consumption selectively reduces the amplitude of the ERN while leaving the $\mathrm{N} 2$ unaffected. In the present research, we tested empirical predictions arising from this simulation work. In so doing, our aim was to provide further insight into the way in which specific cognitive deficits may impact upon the functioning of the MFC. In what follows, we first will describe the conflict-monitoring theory of the ERN and $\mathrm{N} 2$ and then will apply this theory to the findings of Ridderinkhof et al. We then will outline the novel empirical predictions to be tested in the present research.

According to the conflict-monitoring theory, MFC regions detect response conflict - the coactivation of mutually incompatible responses - in order to signal the need for increased cognitive control (Botvinick et al., 1999; Carter et al., 1998). This theory was initially proposed as an account of MFC activity seen during performance of such tasks as the Stroop and flanker tasks. In the flanker task, for example, participants respond to a central target stimulus that is flanked by distracting stimuli that, on some trials, cue the same (congruent) response as the target and, on other trials, cue a different (incongruent) response (Eriksen \& Eriksen, 1974). Increased MFC activity is observed on incongruent trials, apparent in the EEG as an enhanced $\mathrm{N} 2$ component, which the conflict-monitoring theory attributes to the presence of conflict between the differing responses cued by the target and the flanker stimuli.

Subsequent research has used computational-modeling techniques to extend the conflict theory to account for the ERN (Yeung et al., 2004). In simulations using such models (see Figure 1A), it is found that response conflict is increased in the period following errors. This conflict develops when continued stimulus processing after an error leads to activation of the correct response, resulting in con-

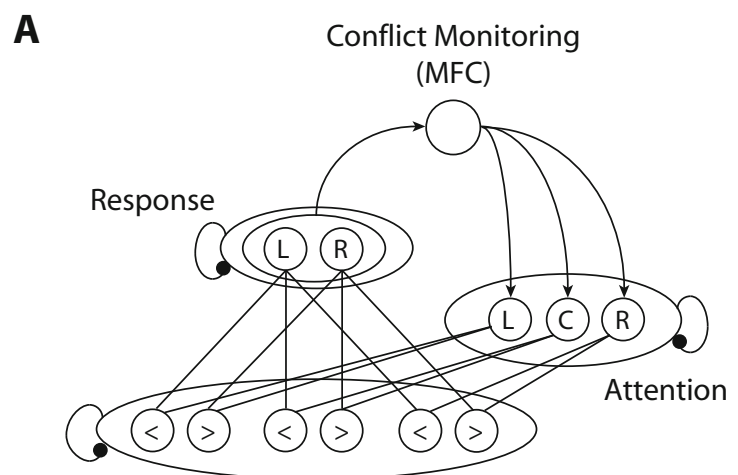

Stimulus

B

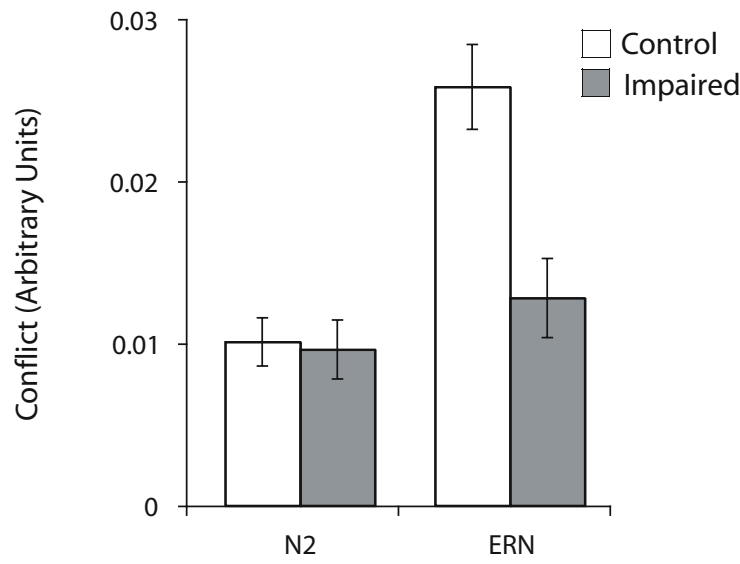

Figure 1. Conflict monitoring and the impact of impaired stimulus processing on medial frontal cortex (MFC) function. (A) Schematic diagram of a model of conflict monitoring in the flanker task. The stimulus layer has units for each possible stimulus (left- and right-pointing target and flanker arrows). Stimulus units feed activity into a response layer with one unit for each possible response. The attention layer has a unit for each of the three locations. The conflict-monitoring system (MFC) is sensitive to co-activation of response units. (B) Results of simulations by Yeung and Cohen (2006), in which combined perceptual and attentional deficits reduced the amplitude of the simulated ERN but did not affect the amplitude of the $\mathbf{N}$, relative to a control condition in which perceptual and attentional processes were intact. 
flict with the incorrect response just produced. The timing of this posterror conflict closely matches the observed timing of the ERN. In this way, the conflict theory provides a unifying account of the ERN and N2: The ERN reflects conflict that develops following errors, whereas the N2 reflects conflict occurring prior to correct responses.

As was mentioned above, Ridderinkhof et al. (2002) found that alcohol consumption leads to a reduction in the amplitude of the ERN but does not affect the N2. This finding presents an apparent challenge to the conflict theory: If we assume that alcohol directly impairs a conflictmonitoring system indexed by the ERN, we would expect alcohol to have a corresponding impact on the N2. However, we have previously argued that the conflict theory can account for Ridderinkhof et al.'s findings if we assume that the primary effect of alcohol in the flanker task is to impair perceptual processing and attentional focus, rather than to disrupt the MFC directly (Yeung \& Cohen, 2006). This interpretation is consistent both with prior research on the effects of alcohol consumption (Colrain et al., 1993; Koelega, 1995) and with results observed in Ridderinkhof et al.'s study. Thus, Ridderinkhof et al. found that alcohol consumption reduced the amplitude of EEG components related to stimulus processing and led to an overall increase in reaction time (RT). As will be discussed further below, this RT increase occurred in the absence of an increase in conflict on incongruent trials.

Using computational simulations of conflict monitoring, we demonstrated that the behavioral and EEG results reported by Ridderinkhof et al. (2002) can be explained in terms of the hypothesis that alcohol consumption causes a combined deficit in perceptual and attentional processing (Yeung \& Cohen, 2006). Thus, the simulation data replicated the observed dissociation between the ERN and the $\mathrm{N} 2$, even though both components were simulated in terms of the detection of response conflict (see Figure 1B). The model was able to account for dissociations between the ERN and the N2 because it holds that these two components are sensitive to different aspects of task processing. In particular, in the model simulations, ERN amplitude depends primarily on processing of the target stimulus (which determines whether correcting responses are produced following errors), whereas N2 amplitude depends critically on processing of the irrelevant flankers (which determines the level of incorrect response activation on correct response trials). Factors that differentially affect the processing of targets and flankers are thus predicted to have dissociable effects on the ERN and the N2.

Critically, the proposed effects of alcohol consumption differentially affect target and flanker processing in just this way. Specifically, whereas perceptual deficits cause impaired processing of both target and flankers, attentional deficits impair processing of the target but actually increase processing of the flankers. Perceptual and attentional deficits therefore have the same negative impact on target stimulus processing (on which the ERN depends) but have opposite, counteracting effects on processing of the distracting flankers (on which the N2 depends). Thus, perceptual and attentional deficits combine to produce a substantial reduction in ERN amplitude but have offsetting effects on the N2. In this way, the conflict theory provides an account of the dissociable effects of alcohol consumption on the ERN and the N2 (see Yeung \& Cohen, 2006, for full details of the simulation methods and results).

The simulation results therefore suggested that Ridderinkhof et al.'s (2002) findings can be explained in terms of alcohol-induced deficits in task processing, rather than as a direct disruption of MFC function. This interpretation led us to a novel prediction: Disrupting perceptual and attentional processes should produce effects on behavior and MFC activity similar to those produced by alcohol consumption in Ridderinkhof et al.'s study. This is the prediction tested here. To this end, we had participants perform a flanker task in which we varied the discriminability of the stimuli. In some blocks, the stimuli were clearly visible, being presented in white against a black background. In other blocks, the stimuli were much less discriminable, being presented in dim gray against a black background. This manipulation of stimulus brightness was designed to produce both hypothesized effects of alcohol consumption: creating perceptual deficits by reducing the strength of stimulus input and inducing attentional deficits by making it more difficult to locate the central target. Thus, our prediction was that these combined effects of dimming the stimuli would affect behavioral and EEG measures in ways that would mimic the effects of alcohol consumption seen in Ridderinkhof et al.'s study.

As compared with the bright stimulus condition, we therefore predicted that dim stimuli would be associated with (1) increased overall RTs, but not increased conflict from incongruent flankers; (2) reduced ERN amplitude; and (3) unchanged N2 amplitude. In testing these predictions, we had two primary goals. The first was to investigate the degree to which MFC function can be influenced in predictable ways by disrupted processing elsewhere in the brain. Findings consistent with our predictions would emphasize the importance of - and the difficulties inherent in - establishing the direction of causality when disturbances of MFC function are seen in association with cognitive or behavioral deficits. Our second goal was to test the specific predictions arising from our earlier simulations of the effects of alcohol consumption on MFC function (Yeung \& Cohen, 2006). Confirmation of our predictions would lend support to the conflict-monitoring theory as an account of normal and disturbed MFC function.

\section{METHOD}

\section{Participants}

There were 14 participants in the experiment, 10 men and 4 women, 18-22 years of age, all recruited from the Carnegie Mellon University community. They received payment or course credit for their involvement. All the participants were right-handed and had normal or corrected-to-normal vision. Informed consent was obtained from each participant at the start of the session.

\section{Procedure}

The participants performed a version of the flanker task in which they responded with a buttonpress to indicate the direction of a central arrow that was surrounded by flanker arrows. There were four stimuli: the congruent stimuli $<<<<<$ and $>>>>>$ and the incongruent stimuli $\langle<\rangle<<$ and $\rangle><>>$. On each 
trial, the participants were first presented with a fixation cross in the center of the screen. The fixation cross disappeared after $400 \mathrm{msec}$, to be followed $100 \mathrm{msec}$ later with an imperative stimulus. The stimulus remained on the screen for $100 \mathrm{msec}$, at which point the screen cleared until $1,500 \mathrm{msec}$ after the participants' response (a buttonpress with their left or right index finger). The fixation cross for the upcoming trial then appeared in the center of the screen. The order in which the four stimuli were presented was randomized, subject to the constraint that each stimulus should appear equally often in each block.

Stimulus brightness was varied across blocks: In half of the blocks, the stimuli were presented in white against a black background (bright stimuli); in the other half of the blocks, the stimuli were presented in dark gray against a black background (dim stimuli). RGB values for the stimuli were as follows: bright $=255$, 255,$255 ; \operatorname{dim}=50,50,50$. Pilot testing established that these RGB values for dim stimuli would produce an RT increase comparable to that seen following alcohol consumption in the study of Ridderinkhof et al. (2002). There were no other differences between blocks of bright and dim stimuli.

The participants first performed separate practice blocks with bright and dim stimuli. They then performed 12 experimental blocks of 68 trials each. Bright and dim stimulus blocks occurred in a repeating $\mathrm{ABBA}$ sequence. Half of the participants began with a bright stimulus block; the other half of the participants began with a dim stimulus block. The participants were given feedback between blocks showing their mean correct RT and error rate, separately for bright and dim stimulus blocks. The participants were instructed to avoid making more errors in dim stimulus blocks than in bright stimulus blocks, just as Ridderinkhof et al. (2002) were careful to instruct their participants to avoid errors following alcohol consumption. The participants were also encouraged to sit in a relaxed position, to minimize eye movement, and to avoid blinking while they performed the task. At a viewing distance of $1 \mathrm{~m}$, each of the arrow stimuli subtended $0.5^{\circ}$ of visual angle vertically and $0.5^{\circ}$ horizontally and were spaced $0.3^{\circ}$ apart.

\section{Electrophysiological Recording and Analysis}

We recorded the $\mathrm{EEG}$, using $\mathrm{Ag} / \mathrm{AgCl}$ electrodes embedded in a fabric cap (Neuroscan, El Paso, TX), from 32 scalp locations arranged according to an extended 10-20 array: FP1, FPz, FP2, F7, F3, Fz, F4, F8, FT7, FC3, FCz, FC4, FT8, T7, C3, Cz, C4, T8, TP7, $\mathrm{CP} 3, \mathrm{CPz}, \mathrm{CP} 4, \mathrm{TP} 8, \mathrm{P} 7, \mathrm{P} 3, \mathrm{Pz}, \mathrm{P} 4, \mathrm{P} 8, \mathrm{POz}, \mathrm{O} 1, \mathrm{Oz}$, and O2. Other electrodes were placed on the right mastoid, above and below the left eye, and on the outer canthi of both eyes. The ground was placed at location AFz. All electrode recordings were referenced to the left mastoid, and electrode impedances were kept below $10 \mathrm{k} \Omega$. EEG data were collected using SynAmps 2 amplifiers (Neuroscan). The data were recorded from 0.1 to $200 \mathrm{~Hz}$, sampled at $1000 \mathrm{~Hz}$ with a resolution of $29.8 \mathrm{nV}$, and amplified with a gain of 2,816.

After recording, the continuous EEG data were corrected for ocular movement artifacts (Semlitsch, Anderer, Schuster, \& Presslich, 1986). We then extracted separate stimulus- and response-locked epochs. Stimulus-locked epochs of $800 \mathrm{msec}(200-\mathrm{msec}$ baseline) were extracted for correct trials only, separately for trials with congruent and incongruent stimuli. Response-locked epochs of $600 \mathrm{msec}$ (200-msec baseline) were extracted separately for trials with correct and incorrect responses. EEG epochs with amplifier saturation or voltage changes greater than $100 \mu \mathrm{V}$ were automatically discarded. Epochs from each channel were then baseline corrected relative to the period from -100 to $0 \mathrm{msec}$ before the event of interest. The data were next bandpass filtered from 0 to $20 \mathrm{~Hz}$, using a two-way least-squares finite impulse response filter with zero phase shift, $3-\mathrm{dB}$ attenuation at cutoff frequencies, and $1-\mathrm{Hz}$ transition bands. The stimulus- and response-locked epochs were then averaged for each participant, separately for the bright and the dim stimulus conditions, prior to quantification of the N2 and ERN components.

The N2 was quantified in correct-trial stimulus-locked averages as the base-to-peak voltage difference between the most negative peak in the window from 200 to $500 \mathrm{msec}$ poststimulus and the immediately preceding positive peak. N2 amplitude was measured for congruent and incongruent trials separately for bright and dim stimulus blocks. The ERN was quantified in response-locked waveforms as the base-to-peak voltage difference between the most negative peak occurring $0-100 \mathrm{msec}$ postresponse and the immediately preceding positive peak. This base-to-peak measure was calculated for correct and error trials, separately for bright and dim stimulus blocks. Consistent with prior studies, the amplitudes of both the ERN and the N2 were maximal at FCz. Our statistical analysis therefore focused on this electrode location. The data were subjected to ANOVAs, using partial eta squared $\left(\eta_{\mathrm{p}}^{2}\right)$ to indicate effect size. Degrees of freedom were corrected using Greenhouse-Geisser epsilon values where appropriate.

\section{RESULTS}

\section{Behavioral Results}

We predicted that impaired stimulus processing in dim stimulus blocks would be reflected in increased RTs, but with no accompanying increase in conflict on incongruent trials. Mean RT and error data relevant to these predictions are given in Table 1. An ANOVA on the RT data revealed reliable main effects of stimulus congruence $\left[F(1,13)=73.5, p<.01, \eta_{\mathrm{p}}^{2}=.85\right]$ and stimulus brightness $\left[F(1,13)=88.7, p<.01, \eta_{\mathrm{p}}^{2}=.87\right]$. Thus, RTs were longer on incongruent trials than on congruent trials and were longer for dim stimuli than for bright stimuli. Critically, however, the effects of stimulus congruence were no greater for dim than for bright stimuli $[F(1,13)=2.67$, $\left.p>.1, \eta_{\mathrm{p}}^{2}=.17\right]$. A corresponding analysis of the error rate data revealed reliable main effects of stimulus congruence $\left[F(1,13)=201.2, p<.01, \eta_{\mathrm{p}}^{2}=.94\right]$ and stimulus brightness $\left[F(1,13)=4.9, p<.05, \eta_{\mathrm{p}}^{2}=.27\right]$, qualified by a reliable interaction between these factors $[F(1,13)=$ $\left.12.2, p<.01, \eta_{\mathrm{p}}^{2}=.48\right]$. This interaction indicated that the effects of stimulus congruence were, in fact, somewhat smaller for dim stimuli than for bright stimuli. Overall, therefore, the data supported our prediction that dim stimuli would be associated with increased RTs, but not with increased interference from incongruent flankers.

Table 1 also presents mean RT for trials with incorrect responses, separately for bright and dim stimuli. An ANOVA comparing these RTs with those on correct trials, averaged across congruent and incongruent trials, revealed a reliable main effect of response type [correct or error; $\left.F(1,13)=225.2, p<.01, \eta_{\mathrm{p}}^{2}=.95\right]$, with RTs on error trials markedly shorter than those on correct responses. There was no reliable interaction between response type

Table 1

Behavioral Results Showing Means and Standard Deviations of Reaction Times (RTs, in Milliseconds) and Error Rates (As Percentages) Across Conditions for Correct Response Trials (Separately for Congruent and Incongruent Stimuli) and for Error Trials in Bright and Dim Stimulus Blocks

\begin{tabular}{|c|c|c|c|c|c|c|c|c|}
\hline \multirow[b]{3}{*}{ Trials } & \multicolumn{4}{|c|}{ Bright } & \multicolumn{4}{|c|}{ Dim } \\
\hline & \multicolumn{2}{|c|}{ RT } & \multicolumn{2}{|c|}{ Errors } & \multicolumn{2}{|c|}{ RT } & \multicolumn{2}{|c|}{ Errors } \\
\hline & $M$ & $S D$ & $M$ & $S D$ & $M$ & $S D$ & $M$ & $S D$ \\
\hline Congruent & 352 & 26 & 3.6 & 2.2 & 381 & 28 & 3.8 & 2.1 \\
\hline Incongruent & 419 & 48 & 27.8 & 5.9 & 442 & 48 & 23.4 & 6.0 \\
\hline Error & 329 & 34 & - & - & 351 & 33 & - & - \\
\hline
\end{tabular}


and stimulus brightness $\left[F(1,13)=1.4, p>.25, \eta_{\mathrm{p}}^{2}=.10\right]$. A subsequent pairwise comparison revealed that error RTs were reliably longer in dim stimulus blocks than in bright stimulus blocks $\left[t(13)=3.3, p<.01, \eta_{\mathrm{p}}^{2}=.46\right]$.

In subsequent analyses, we investigated whether stimulus brightness would affect the participants' ability to adjust their behavior following incorrect responses. It has previously been reported that participants may respond more slowly (Rabbitt, 1966), and with less interference from distracting flankers (Ridderinkhof et al., 2002), on the trial immediately following an error. Ridderinkhof et al. found that alcohol consumption abolished at least the latter effect (they did not report posterror slowing effects). We were therefore interested in whether sequential adjustment effects would be similarly sensitive to variations in stimulus brightness. However, we did not observe sequential adjustment effects in either condition of our experiment: Our participants responded as quickly following errors as following correct responses (393 vs. $394 \mathrm{msec} ; F<1$ ) and suffered similar levels of incongruent-trial interference following errors and following correct responses (60 vs. $64 \mathrm{msec} ; F<1$ ). The failure to observe any sequential adjustment effects in our study, even in the bright stimulus condition, makes it impossible to compare this aspect of our results with those of Ridderinkhof et al.

\section{EEG Results}

As was described above, the behavioral effects of stimulus brightness were consistent with our predictions, showing an overall increase in RTs but no increase in flanker interference. These behavioral findings suggest that our manipulation of stimulus brightness was effective in pro- ducing a combined perceptual and attentional deficit. Of interest, then, are the corresponding effects of stimulus brightness on the ERN and N2: Our prediction was that dimming the stimuli would cause a selective reduction in ERN amplitude while leaving the N2 intact, consistent with the effects of alcohol consumption observed by Ridderinkhof et al. (2002).

Grand-averaged stimulus- and response-locked waveforms from electrode $\mathrm{FCz}$ are plotted in Figure 2. The upper panel of Figure 2 plots stimulus-locked waveforms from congruent and incongruent trials, separately for bright and dim stimuli. The N2 is clearly evident as a negative peak, occurring $\sim 300 \mathrm{msec}$ after stimulus onset, that is enhanced on trials with incongruent stimuli. An ANOVA on N2 amplitudes revealed a significant main effect of stimulus congruence $[F(1,13)=6.4, p<.05$, $\left.\eta_{\mathrm{p}}^{2}=.33\right]$, with greater $\mathrm{N} 2$ amplitude on incongruent trials than on congruent trials. However, there was no reliable main effect of stimulus brightness $[F(1,13)=3.4$, $\left.p>.05, \eta_{\mathrm{p}}^{2}=.21\right]$ nor any significant interaction between brightness and congruence $(F<1)$. The null interaction indicates that, as was predicted, N2 amplitude did not differ reliably between bright and dim stimulus conditions. These results are summarized in Figure 3, which plots N2 amplitude (calculated as the difference in peak amplitude between incongruent and congruent trials) as a function of stimulus brightness.

The lower panel of Figure 2 plots grand-averaged response-locked waveforms for correct and error trials, separately for bright and dim stimuli. The ERN is evident as a large amplitude negative peak occurring $\sim 50 \mathrm{msec}$ after incorrect responses. This peak is largely absent fol-
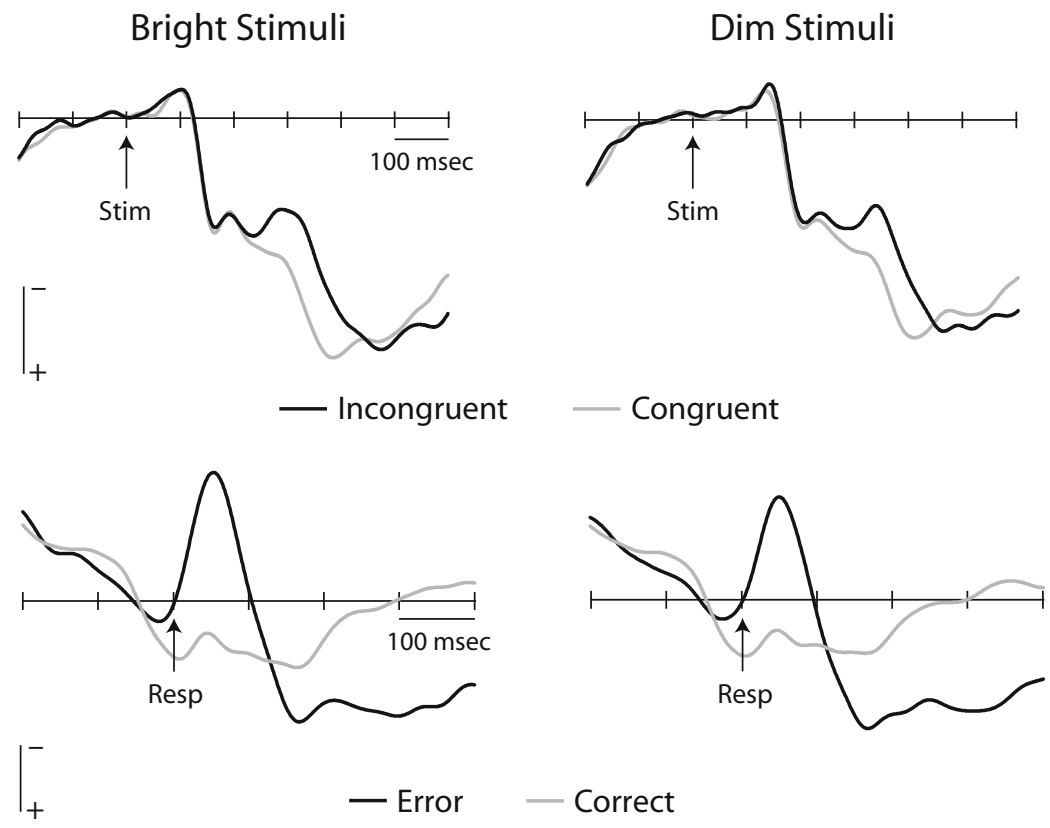

Figure 2. Grand-averaged ERP waveforms at electrode FCz. Top panels: Stimuluslocked waveforms for correct trials with congruent and incongruent stimuli, separately for bright and dim stimulus blocks. Bottom panels: Response-locked waveforms for correct and error trials, separately for bright and dim stimulus blocks. The vertical scale bars indicate a $5-\mu \mathrm{V}$ increment in both panels. 
lowing correct responses. An ANOVA on the amplitude of the postresponse negative peak revealed a main effect of response type [correct or error; $F(1,13)=68.1, p<.01$, $\left.\eta_{\mathrm{p}}^{2}=.84\right]$ but no reliable main effect of stimulus brightness $\left[F(1,13)=2.3, p>.1, \eta_{\mathrm{p}}^{2}=.15\right]$. Critically, there was a reliable interaction between these two factors $[F(1,13)=$ $\left.5.5, p<.05, \eta_{\mathrm{p}}^{2}=.30\right]$, reflecting the predicted reduction in ERN amplitude in dim stimulus blocks relative to bright stimulus blocks. These results are summarized in Figure 3, which plots ERN amplitude (calculated as the difference in peak amplitude between error and correct trials) separately for bright and dim stimulus blocks.

A final set of analyses focused on the latency of the N2 and ERN peaks. Although not a focus of our a priori predictions, an increase in N2 latency in the dim stimulus condition was apparent in the ERP waveforms plotted in Figure 2. Such an increase would be consistent with our earlier computational simulations (Yeung \& Cohen, 2006), in which the combined perceptual and attentional deficits resulted in a prolongation of response conflict (but no increase in its peak amplitude). With N2 latency quantified as the time point of greatest difference between incongruent and congruent trial ERP waveforms at electrode $\mathrm{FCz}$, we found a reliable increase in latency in the dim stimulus condition, as compared with the bright stimulus condition $\left[t(13)=6.9, p<.01, \eta_{\mathrm{p}}^{2}=.79\right]$. A corresponding analysis of ERN latency revealed no reliable effect of stimulus brightness $(t<1)$, again consistent with the results of our computational simulations (Yeung \& Cohen, 2006).

Taken together, the EEG data support the predictions of the conflict-monitoring theory, as derived from our

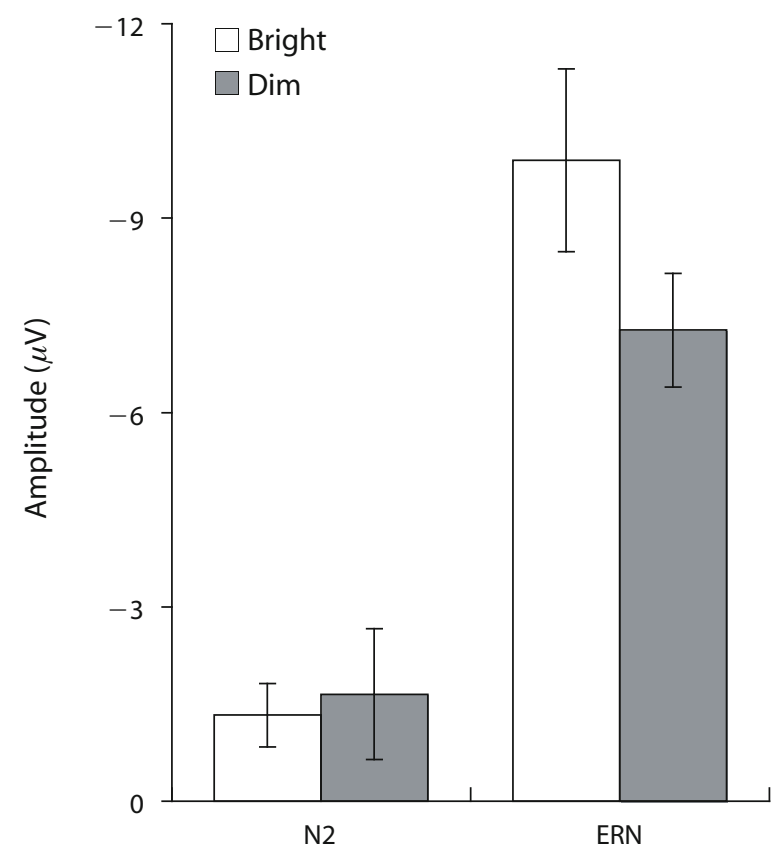

Figure 3. Average N2 and ERN amplitudes at electrode $\mathrm{FCz}$ for bright and dim stimulus blocks. $\mathbf{N} 2$ amplitude is measured in terms of differences between congruent and incongruent trials. ERN amplitude is measured in terms of differences between correct and error trials. earlier computational-modeling work: The presentation of dim stimuli resulted in a selective reduction in ERN amplitude, while leaving N2 amplitude unaffected. These results match the predicted effects of a combined perceptual and attentional deficit (compare Figures 1B and 3), and they mimic the qualitative pattern of effects seen in Ridderinkhof et al.'s (2002) study of the effects of alcohol consumption on MFC function.

\section{DISCUSSION}

On the basis of the conflict-monitoring theory of MFC function, we generated novel predictions about the impact of presenting dim (low-contrast) stimuli. These predictions were borne out in our behavioral and EEG data. In what follows, we will consider the implications of our findings in relation to our two primary goals: evaluating predictions of the conflict-monitoring theory of MFC function arising from our earlier simulations of alcohol effects, and investigating how MFC function may be influenced in predictable ways by disrupted processing elsewhere in the brain.

In our prior computational-modeling research, we demonstrated that dissociations between the ERN and the N2 could arise from a combined deficit in perceptual and attentional processing (Yeung \& Cohen, 2006). According to the conflict-monitoring theory, the ERN is generated when continued processing of the stimulus after an error leads to the activation of the correct response (which then conflicts with the incorrect response just produced). ERN amplitude should, therefore, be affected in the same way by perceptual and attentional deficits, both of which reduce the likelihood of correctly identifying the target - and hence, the likelihood of activating the correct response-following an initial error. Thus, both deficits tend to reduce the amplitude of the ERN. In contrast, the conflict-monitoring theory predicts that the N2-which is proposed to reflect flanker-induced conflict-should be affected in counteracting ways by perceptual and attentional deficits: Whereas perceptual deficits reduce the strength of flanker processing, reduced attentional focus will have the opposite effect. As a consequence, the effects of the two deficits offset one another to leave N2 amplitude unchanged.

The present manipulation of stimulus brightness was designed to recreate both perceptual deficits (by reducing stimulus strength) and attentional impairments (by increasing the difficulty of locating the central target). As was described above, the results of this manipulation were consistent with the predictions of the conflict-monitoring theory: Behaviorally, presenting dim stimuli resulted in an overall increase in RT but did not cause an increase in the interfering effects of incongruent flanker stimuli. Indeed, the effects of flanker interference were, if anything, somewhat smaller in dim stimulus blocks than in bright stimulus blocks. In the EEG data, presenting dim stimuli led to a reduction in ERN amplitude but no change in the amplitude of the N2. Taken together, these results demonstrate that the conflict-monitoring theory can provide an integrative account of MFC function as it is reflected in 
the ERN and N2, including providing a principled account of dissociations between these two components.

The observed reduction in ERN amplitude suggests that dimming the stimuli did more than simply delay the onset of task processing. The presence of a processing delay could explain the observed increase in RT and the corresponding increase in N2 peak latency. However, if processing was simply delayed but otherwise proceeded unchanged from bright stimulus trials, ERN amplitude should not change. Thus, processing delay cannot easily explain why ERN amplitude was reduced with dim stimuli (particularly given that error rates did not increase). This hypothesis also fails to explain why the onset of the N2 occurred at similar latencies in bright and dim stimulus conditions: As is shown in Figure 2, N2 differences between congruent and incongruent trials began to emerge at around the same time, $220-230 \mathrm{msec}$ poststimulus, in the two conditions. Taken together, these results support our interpretation that reducing stimulus contrast influenced the dynamics of task processing, rather than merely delaying its onset.

Our findings regarding the ERN are consistent with the results of prior studies demonstrating that ERN amplitude tends to be reduced as task difficulty increases (e.g., Holroyd \& Coles, 2002; Scheffers \& Coles, 2000). However, in this respect, our findings contrast with those in Leuthold and Sommer (1999) and Elton, Spaan, and Ridderinkhof (2004). Both of these studies showed ERN amplitude to be unaffected when task difficulty and error rate were increased by increasing the perceptual similarity among the stimuli to be discriminated. The results of these studies not only contrast with our findings, but also present a challenge to all existing theories of the ERNthe conflict theory included - because all current theories propose that the ERN depends on deriving an accurate representation of the target stimulus or the correct response. Increasing the difficulty of deriving this representation should therefore reduce ERN amplitude.

One potentially relevant feature of the designs used by Leuthold and Sommer (1999) and Elton et al. (2004) is that error rates were very low $(<5 \%)$ in the easier versions of their tasks. It is possible that these tasks were sufficiently easy that errors most often occurred when the participants' attention wandered from the task, so that on these trials the participants failed to derive a useful representation of the stimulus and required response. Such errors would therefore be expected to elicit little or no ERN. In contrast, in the more difficult condition, a larger proportion of errors might occur because of premature responding (cf. Scheffers \& Coles, 2000), so that further processing of the stimulus could lead to activation of the correct response (and hence, a large ERN). Thus, the typical effects of task difficulty might be reduced or absent if the primary cause of incorrect responding differed across conditions. This account is clearly post hoc, but its central prediction - of a nonmonotonic relationship between ERN amplitude and task difficulty — could be tested empirically in future research.

Another key issue for future research is the relationship between the effects of stimulus brightness and the effects of alcohol consumption. As we have discussed at length, the present research was inspired by our prior computational-modeling work on the effects of alcohol consumption (Yeung \& Cohen, 2006). That research demonstrated that a combined perceptual and attentional deficit could account for alcohol effects on behavioral and EEG measures reported by Ridderinkhof et al. (2002). The present research showed that qualitatively similar effects can be recreated by manipulating stimulus brightness. Thus, our results are consistent with the hypothesis that the effects of alcohol consumption on MFC function are mediated indirectly, through perceptual and attentional impairments. However, it remains possible that stimulus brightness and alcohol consumption affect MFC function in quite different ways even though their behavioral and EEG signatures are superficially similar. Further work is required to distinguish between these alternative accounts.

In this context, it would be particularly informative to perform a direct experimental comparison between the effects of alcohol consumption and the effects of impaired stimulus processing. Specifically, one might conduct a study to measure ERN and N2 amplitude in two conditions: one in which the participants have consumed alcohol, and a second in which the participants are sober but in which they are presented with degraded stimuli, so that their behavioral performance matches that seen in the alcohol condition. Of interest would be whether alcohol consumption affects MFC function (as reflected in the ERN and N2) above and beyond the effects of stimulus degradation. In this way, one could extend the present methods to provide further insight into the effects of alcohol consumption on cognitive processing.

Such an approach would also permit investigation of some apparent discrepancies between our results and those of Ridderinkhof et al. (2002). Three issues in particular might be addressed. First, as was noted above, we did not observe reliable sequential adjustment effects in the present study, and we were therefore unable to compare this aspect of our results with those of Ridderinkhof et al. (who observed reliable sequential adjustments in their placebo condition). A possible cause of this discrepancy is that our experiment involved far fewer trials (816) than did the study of Ridderinkhof et al. (whose participants performed three sessions of 1,760 trials). It could be that our participants were able to maintain a high, stable level of performance and concentration throughout the experiment because the experiment was relatively short.

The second discrepancy concerns our finding that N2 latency increased in the dim stimulus condition, whereas Ridderinkhof et al. (2002) did not report a corresponding effect of alcohol consumption. However, it is notable that the morphology of our ERP waveforms was quite different from that seen in Ridderinkhof et al.'s study. In particular, because of temporal overlap between the N2 and P300, in Ridderinkhof et al.'s data the N2 peak did not coincide with the amplitude of the peak difference between congruent and incongruent trials. It is this difference that is critical to the predictions of the conflict theory, and it was the latency of this difference that was affected by stimulus brightness in the present study (recall that the latency of $\mathrm{N} 2$ onset 
did not differ across conditions). A detailed inspection of Ridderinkhof et al.'s results (their Figure 1) suggests that the peak difference between congruent and incongruent trials was somewhat delayed following alcohol consumption, an effect that would be consistent with the present findings. Nonetheless, a direct comparison between morphologically similar ERP waveforms would provide the most informative contrast between the respective effects of alcohol consumption and stimulus degradation.

The final issue to address would be our finding of a slightly reduced error rate in the dim stimulus condition, which contrasts with the matched error rates between placebo and alcohol conditions in Ridderinkhof et al.'s (2002) study. The reduced error rate we observed in dim stimulus blocks was most likely a consequence of the fact that our instructions emphasized accuracy in these blocks; without these instructions, participants would tend to make more errors, not fewer, in this condition. However, a direct contrast between the effects of alcohol consumption and stimulus degradation would require a close match between the behavioral consequences of each manipulation. In this context, it is noteworthy that ERN amplitude has typically been found to vary inversely with error rate (e.g., Falkenstein et al., 1990; Gehring et al., 1993). Given this, the unexpected decrease in error rate in the dim stimulus condition would, if anything, tend to counteract the predicted (and observed) reduction in ERN amplitude in this condition. Thus, although the error rate difference complicates a direct comparison between the effects of alcohol and stimulus degradation, it does not call into doubt our primary finding, which is that dimming the stimuli led to a substantial reduction in ERN amplitude.

These issues notwithstanding, a key implication of our findings is that MFC function is critically sensitive to disruptions in processing elsewhere in the brain. In particular, we have shown that by varying stimulus brightness-a manipulation designed to affect perceptual and attentional processes - we can influence MFC function in systematic and predictable ways. In this regard, our findings have broader implications for the body of research, reviewed above, in which the ERN and N2 are used to probe the nature of cognitive deficits observed in clinical populations and following the administration of various drugs. Where disruptions of MFC function are observed in these contexts, it is tempting to infer that the MFC disturbance plays a direct causal role. However, the present study suggests the need for caution when drawing these kinds of causal inference: The observed disruption of MFC function may, in fact, be a secondary consequence of primary damage to other systems with which the MFC interacts. In such cases, clinical treatments or remedial strategies targeted at MFC functioning may prove ineffective.

It is particularly difficult to establish whether MFC disruptions play a causal role when experimental groups differ significantly in their behavioral performance, because the output of MFC monitoring systems will, by definition, be affected by any change in task processing and task performance. This line of reasoning suggests that performance-matched controls - as suggested above in the proposed study of alcohol effects - may be helpful more generally in establishing the contribution of MFC disturbances to observed cognitive deficits. The notion is that a control group could be given a modified version of the experimental task that has been designed appropriately to match performance across groups. To the degree that differences in MFC function continue to be observed in the critical experimental group, even after behavioral performance is matched in this way, one could more securely infer that MFC function is indeed directly disturbed in that group. With this augmented approach, the ERN and N2 could provide deeper insight into the way in which disturbed MFC functioning contributes to the cognitive deficits observed in various clinical populations.

A final implication of the present research, when taken together with our earlier computational work (Yeung \& Cohen, 2006), is that formal models may provide a crucial tool for investigating the role of the MFC in performance monitoring and cognitive control. Because the normal functioning of the MFC depends critically on its interactions with a complex network of brain regions, disturbances to individual parts of that network may have subtle and unexpected consequences for the workings of the system as a whole. In the present context, for example, it is not immediately obvious that presenting dim stimuli should selectively affect one expression of MFC function (the ERN) while leaving another (the N2) unaffected. Nor is it obvious that these effects should mimic the consequences of alcohol consumption. However, computational modeling provided a framework within which we could articulate these theoretical claims, and provided the basis for generating the novel, testable predictions that were addressed in the present study.

\section{AUTHOR NOTE}

This research was supported by the National Institutes of Health Conte Center for Neuroscience Research (Grant P50-MH62196 to N.Y.) and by the Netherlands Organization for Scientific Research (to S.N.). The authors thank Nisan Mol for his help with pilot work. Correspondence concerning this article should be addressed to N. Yeung, Department of Experimental Psychology, University of Oxford, South Parks Road, Oxford OX1 3UD, England (e-mail: nicholas.yeung@psy.ox.ac.uk).

\section{REFERENCES}

Alain, C., McNeely, H. E., He, Y., Christensen, B. K., \& West, R. (2002). Neurophysiological evidence of error-monitoring deficits in patients with schizophrenia. Cerebral Cortex, 12, 840-846.

Botvinick, M. M., Nystrom, L. E., Fissell, K., Carter, C. S., \& CoHEN, J. D. (1999). Conflict monitoring versus selection-for-action in anterior cingulate cortex. Nature, 402, 179-181.

Carter, C. S., Braver, T. S., Barch, D. M., Botvinick, M. M., Noll, D., \& Cohen, J. D. (1998). Anterior cingulate cortex, error detection, and the online monitoring of performance. Science, 280, 747-749.

Colrain, I. M., Taylor, J., Mclean, S., Buttery, R., Wise, G., \& Montgomery, I. (1993). Dose dependent effects of alcohol on visual evoked potentials. Psychopharmacology, 112, 383-388.

Croxson, P. L., Johansen-Berg, H., Behrens, T. E., Robson, M. D., PinsK, M. A., Gross, C. G., ET AL. (2005). Quantitative investigation of connections of the prefrontal cortex in the human and macaque using probabilistic diffusion tractography. Journal of Neuroscience, 25, 8854-8866.

Curtin, J. J., \& FAirchild, B. A. (2003). Alcohol and cognitive control: Implications for regulation of behavior during response conflict. Journal of Abnormal Psychology, 112, 424-436. 
de Bruijn, E. R., Hulstijn, W., Verkes, R. J., Ruigt, G. S., \& Sabbe, B. G. (2004). Drug-induced stimulation and suppression of action monitoring in healthy volunteers. Psychopharmacology, 177, 151-160.

Elton, M., Spaan, M., \& Ridderinkhof, K. R., (2004). Why do we produce errors of commission? An ERP study of stimulus deviance detection and error monitoring in a choice go/no-go task. European Journal of Neuroscience, 20, 1960-1968.

EriKsen, B. A., \& Eriksen, C. W. (1974). Effects of noise letters upon the identification of a target letters in a nonsearch task. Perception \& Psychophysics, 16, 143-149.

Falkenstein, M., Hohnsbein, J., Hoorman, J., \& Blanke, L. (1990). Effects of errors in choice reaction tasks on the ERP under focused and divided attention. In C. H. M. Brunia, A. W. K. Gaillard, \& A. Kok (Eds.), Psychophysiological brain research (Vol. 1, pp. 192-195). Tilburg, The Netherlands: Tilburg University Press.

Gehring, W. J., Goss, B., Coles, M. G. H., Meyer, D. E., \& DonCHIN, E. (1993). A neural system for error detection and compensation. Psychological Science, 4, 385-390.

Gehring, W. J., Himle, J., \& Nisenson, L. G. (2000). Action-monitoring dysfunction in obsessive-compulsive disorder. Psychological Science, 11, 1-6.

Gehring, W. J., \& Knight, R. T. (2000). Prefrontal-cingulate interactions in action monitoring. Nature Neuroscience, 3, 516-520.

HaJCAK, G., McDonald, N., \& Simons, R. F. (2003). Anxiety and errorrelated brain activity. Biological Psychology, 64, 77-90.

HaJCaK, G., McDonald, N., \& Simons, R. F. (2004). Error-related psychophysiology and negative affect. Brain \& Cognition, 56, 189-197.

Holroyd, C. B., \& Coles, M. G. H. (2002). The neural basis of human error processing: Reinforcement learning, dopamine, and the errorrelated negativity. Psychological Review, 109, 679-709.

Koelega, H. S. (1995). Alcohol and vigilance performance: A review. Psychopharmacology, 118, 233-249.

KopP, B., \& RisT, F. (1999). An event-related brain potential substrate of disturbed response monitoring in paranoid schizophrenic patients. Journal of Abnormal Psychology, 108, 337-346.

Kopp, B., Rist, F., \& Mattler, U. (1996). N200 in the flanker task as a neurobehavioral tool for investigating executive control. Psychophysiology, 33, 282-294.

LEUTHOLD, H., \& SOMmer, W. (1999). ERP correlates of error processing in spatial S-R compatibility tasks. Clinical Neurophysiology, 110, 342-357.

LuU, P., Collins, P., \& Tucker, D. M. (2000). Mood, personality and self-monitoring: Negative affect and emotionality in relation to fronta lobe mechanisms of error monitoring. Journal of Experimental Psychology: General, 129, 43-60.

McNeely, H. E., West, R., Christensen, B. K., \& Alain, C. (2003). Neurophysiological evidence for disturbances of conflict processing in patients with schizophrenia. Journal of Abnormal Psychology, 112, 679-688.

Nieuwenhuis, S., Nielen, M. M., Mol, N., Hajcak, G., \& VeltMAN, D. J. (2005). Performance monitoring in obsessive-compulsive disorder. Psychiatry Research, 134, 111-122.

Nieuwenhuis, S., Yeung, N., van den Wildenberg, W., \& Rid-
DERINKHOF, K. R. (2003). Electrophysiological correlates of anterior cingulate function in a go/no-go task: Effects of response conflict and trial type frequency. Cognitive, Affective, \& Behavioral Neuroscience, 3, 17-26.

RABBitT, P. M. A. (1966). Errors and error correction in choice-response tasks. Journal of Experimental Psychology, 71, 264-272.

Riba, J., Rodriguez-Fornells, A., Morte, A., Munte, T. F., \& BarBANOJ, M. J. (2005). Noradrenergic stimulation enhances human action monitoring. Journal of Neuroscience, 25, 4370-4374.

Ridderinkhof, K. R., de Vlugt, Y., Bramlage, A., Spaan, M., Elton, M., Snel, J., \& BAND, G. P. H. (2002). Alcohol consumption impairs detection of performance errors in mediofrontal cortex. Science, 298, 2209-2211.

Ruchsow, M., Walter, H., Buchheim, A., Martius, P., Spitzer, M., KACHELE, H., ET AL. (2006). Electrophysiological correlates of error processing in borderline personality disorder. Biological Psychology, 72, 133-140.

Scheffers, M. K., \& Coles, M. G. H. (2000). Performance monitoring in a confusing world: Error-related brain activity, judgements of response accuracy, and types of errors. Journal of Experimental Psychology: Human Perception \& Performance, 26, 141-151.

Semlitsch, H. V., Anderer, P., Schuster, P., \& Presslich, O. (1986). A solution for reliable and valid reduction of ocular artifacts, applied to the P300 ERP. Psychophysiology, 23, 695-703.

Tieges, Z., Ridderinkhof, K. R., Snel, J., \& KoK, A. (2004). Caffeine strengthens action monitoring: Evidence from the error-related negativity. Cognitive Brain Research, 21, 87-93.

Ullsperger, M., \& vON CRAMON, D. Y. (2006). The role of intact frontostriatal circuits in error processing. Journal of Cognitive Neuroscience, 18, 651-664.

Ullsperger, M., von Cramon, D. Y., \& Muller, N. G. (2002). Interactions of focal cortical lesions with error processing: Evidence from event-related brain potentials. Neuropsychology, 16, 548-561.

van Hoesen, G. W., Morecraft, R. J., \& Vogt, B. A. (1993). Connections of the monkey cingulate cortex. In B. A. Vogt \& M. Gabriel (Eds.), Neurobiology of cingulate cortex and limbic thalamus: A comprehensive handbook (pp. 249-284). Boston: Birkhäuser.

van Veen, V., \& Carter, C. S. (2002). The timing of action monitoring in rostral and caudal anterior cingulate cortex. Journal of Cognitive Neuroscience, 14, 593-602.

Yeung, N., Botvinick, M. M., \& Cohen, J. D. (2004). The neural basis of error detection: Conflict monitoring and the error-related negativity. Psychological Review, 111, 931-959.

Yeung, N., \& Cohen, J. D. (2006). The impact of cognitive deficits on conflict monitoring: Predictable dissociations between the errorrelated negativity and N2. Psychological Science, 17, 164-171.

Zirnheld, P. J., Carroll, C. A., Kieffaber, P. D., O'Donnell, B. F., SheKhar, A., \& Hetrick, W. P. (2004). Haloperidol impairs learning and error-related negativity in humans. Journal of Cognitive Neuroscience, 16, 1098-1112.

(Manuscript received December 30, 2006; revision accepted for publication June 18, 2007.) 